\title{
Classic medulloblastoma
}

INSERM

\section{Source}

INSERM. (1999). Orphanet: an online rare disease and orphan drug data base. Classic medulloblastoma. ORPHA:251867

Classic medulloblastoma is a histological variant of medulloblastoma (see this term), an embryonic malignancy, having a midline location, occurring most often in children and manifesting with variable symptoms such as headaches, nausea, vomiting and ataxia. 